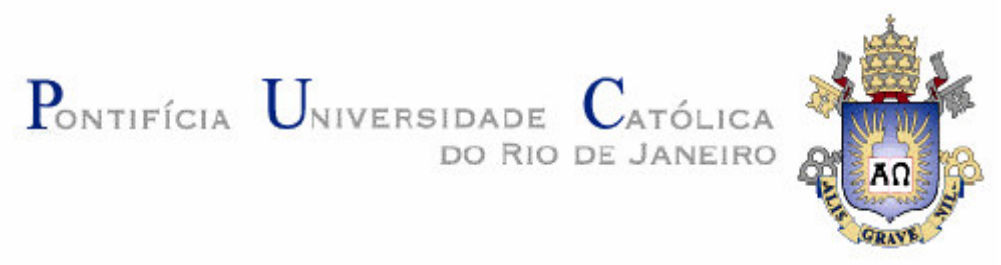

Guilherme França dos Santos Paiva

\title{
LETRAMENTO FUNCIONAL FRENTE À ROTULAGEM NUTRICIONAL DE ALIMENTOS.
}

Dissertação de Mestrado

Dissertação apresentada como requisito parcial para obtenção do grau de Mestre pelo Programa de Pós-Graduação em Administração de Empresas do Departamento de Administração da PUC-Rio.

Orientador: Prof. Paulo César Motta

Rio de Janeiro

Abril de 2010 


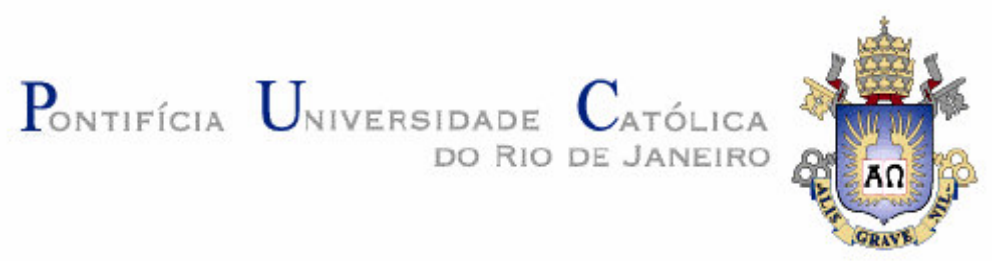

Guilherme França dos Santos Paiva

\author{
Letramento Funcional Frente à \\ Rotulagem Nutricional de Alimentos.
}

Dissertação apresentada como requisito parcial para obtenção do grau de Mestre pelo Programa de Pós-Graduação em Administração de Empresas da PUC-Rio. Aprovada pela Comissão Examinadora abaixo assinada.

Prof. Paulo César Motta Orientador Departamento de Administração - PUC-Rio

Prof ${ }^{a}$. Maria do Carmo Leite de Oliveira Departamento de Letras-PUC-Rio

Prof. Paulo Vicente dos Santos Alves ESPM-RJ

Profa. Mônica Herz

Vice-Decana de Pós-Graduação do CCS

Rio de Janeiro, 13 de abril de 2010 
Todos os direitos reservados. É proibida a reprodução total ou parcial do trabalho sem autorização da universidade, do autor e do orientador.

\section{Guilherme França dos Santos Paiva}

Graduou-se em Comunicação Social/ Publicidade e Propaganda na Universidade Federal do Rio de Janeiro em 2005. Trabalhou na área de comunicação e criação em agências de propaganda, também na área de marketing em empresas de consultoria e treinamento.

Ficha Catalográfica

CDD: 658 


\section{Agradecimentos}

Ao meu orientador Professor Paulo César Motta, pelo estímulo, presença, paciência e confiança para a realização deste trabalho.

À Coordenação de Aperfeiçoamento de Pessoal de Nível Superior (CAPES), por ter financiado meus estudos.

A meus pais, irmãos e cunhados, pelo total apoio, envolvimento, paciência e compreensão em dois anos de estudo.

Aos amigos Danilo Arruda e Mariana Cupolillo, que contribuíram com suas opiniões em diversas fases do trabalho.

Aos amigos Fabio Silva, Enderson França, Luciana Velloso, Rodrigo Viégas, Renata Paiva, Monique Sampaio, Leandro Miranda e Joana Costa pelo auxílio dado na obtenção da amostra necessária.

Ao amigo Renato Almeida, pela ajuda técnica na organização dos dados coletados.

Aos amigos Rodrigo Viégas, Renata Paiva e Luciana Velloso, pelo auxílio na normatização.

A todos os familiares pelo total apoio, paciência e compreensão.

Aos demais amigos e colegas, pelo apoio. 


\section{Resumo}

Paiva, Guilherme França dos Santos; Motta, Paulo Cesar (orientador). Letramento funcional frente à rotulagem nutricional de alimentos. Rio de Janeiro, 2010. 115p. Dissertação de Mestrado - Departamento de Administração, Pontifícia Universidade Católica do Rio de Janeiro.

O presente estudo tem como objetivo avaliar o nível de entendimento dos consumidores aplicado à rotulagem nutricional de alimentos industrializados. Ele suporta a idéia de que cada consumidor funciona de maneira distinta frente ao contexto de análise dos rótulos nutricionais. Através de uma pesquisa empírica que utiliza a metodologia de um sistema de avaliação de letramento funcional, traça-se o perfil dos consumidores e mensura-se a pontuação dos entrevistados frente ao conhecimento de aspectos da rotulagem nutricional aplicada pela Agência Nacional de Vigilância Sanitária. Os resultados mostram evidências de que existem consumidores desconhecedores de elementos nutricionais básicos, outros incapazes de desenvolver tarefas simples de análise de rótulos. Níveis diferentes de entendimento frente as variáveis sexo, renda, escolaridade, idade, leitura de rótulos, exercícios, prática de refeições fora de casa e compra de alimentos para a família revelam realidades heterogêneas de escolhas alimentares, trazendo subsídios importantes para a área de comportamento do consumidor e de políticas públicas.

\section{Palavras-chave}

Letramento funcional; decisão de compra; comportamento do consumidor; rotulagem nutricional. 


\section{Abstract}

Paiva, Guilherme França dos Santos; Motta, Paulo Cesar (advisor). Functional Literacy and Food Nutrition Labeling. Rio de Janeiro, 2010. 115p. MSc Dissertation - Departamento de Administração, Pontifícia Universidade Católica do Rio de Janeiro

This study aims to evaluate the level of understanding of consumers in the context of food nutrition labeling. It supports the idea that each consumer works differently against the background of the analysis of nutrition labels. Through an empirical research that uses the methodology of an assessment system for functional literacy, a profile of consumers is drawn and a score of the respondents related to nutrition label aspects knowledge applied by Anvisa is measured. Results show evidence that consumers are unaware of basic nutritional elements, unable to develop other simple tasks of label analysis. Different levels of understanding by the variables gender, level of income, level of education, age, reading labels habit, exercise habit, practice of eating out and buying food for the family reveal heterogeneous realities of food choices, bringing important benefits to the area of consumer behavior and public policy.

\section{Keywords}

Functional literacy; purchase decision; consumer behavior; nutritional label. 


\section{Sumário}

$\begin{array}{ll}\text { 1. Introdução } & 10\end{array}$

1.2. Relevância 11

1.2. Objetivos 12

$\begin{array}{ll}\text { 1.3. Hipóteses de Pesquisa } & 13\end{array}$

1.4. Estrutura da Dissertação 15

1.5. Definição de Termos 16

2. Revisão da Literatura 18

$\begin{array}{ll}\text { 2.1. O Conceito de Letramento } & 18\end{array}$

2.1.1. A Dimensão Individual do Letramento 18

2.1.2. A Dimensão Social do Letramento 19

2.1.2.1. Tendências Liberais e Radicais da Dimensão Social 19

$\begin{array}{lr}\text { 2.1.3. Numeramento } & 20\end{array}$

2.1.4. Letramento do Consumidor 21

2.1.5. Impacto do Letramento no Consumo 22

2.2. Sistemas de Avaliação Educacionais 24

$\begin{array}{ll}\text { 2.3. Rótulo } & 27\end{array}$

2.3.1. Origens da Legislação Brasileira sobre Rótulos 27

2.3.2. Evolução da Rotulagem de Alimentos no Brasil 28

2.3.3. Principais Leis de Rotulagem Nutricional 30

2.3.4. Funções do Rótulo 32

3. Método 35

4. Dados Gerais da Amostra 41 
5. Resultados 44

5.1. Análise da Qualidade da Prova 44

5.2. Níveis de Entendimento por Variáveis de Perfil 49

5.2.1. Sexo 50

5.2.2. Hábito de Leitura de Rótulos 51

5.2.3. Hábito de Compra de Alimentos para a Família 52

5.2.4. Escolaridade 54

5.2.5. Renda Familiar Mensal Bruta 55

5.2.6. Hábito de Realizar Refeições Fora de Casa Dia de Semana 55

5.2.7. Hábito de se Fazer Exercícios 56

$\begin{array}{ll}\text { 5.2.8. Idade } & 57\end{array}$

5.3. Nível de Entendimento por Questão 58

6. Conclusão 80

6.1. Implicações Gerenciais 80

6.2. Sugestão para Estudos Futuros 82

7. Referências Bibliográficas $\quad 84$

Anexo 1: Glossário do Questionário 94

Anexo 2: Qualidade da Prova e Distribuição de Freqüência 102

Anexo 3: Análise Frente à Variável Sexo 106

Anexo 4: Análise Frente a Consulta a Rótulos 107

Anexo 5: Análise Frente a Compra de Alimentos para a Família 108

Anexo 6: Análise Frente a Escolaridades Distintas 109

Anexo 7: Análise Frente a Escolaridades Distintas 110

Anexo 8: Análise Hábito de Realizar Refeições Fora de Casa 111

Anexo 9: Análise Frente a Hábito de se Fazer Exercícios 112

Anexo 10: Questionário 113 
SIGLAS

ABIAD - Associação Brasileira das Indústrias de Alimentos Dietéticos

Anvisa - Agência Nacional de Vigilância Sanitária

CNNPA - Comissão Nacional de Normas e Padrões para Alimentos

CTCAF - Comissão de Assessoramento Técnico-Científico em Alimentos

Funcionais e Novos Alimentos

ENDEF - Estudo Nacional de Despesas Familiares

ENEM - Exame Nacional do Ensino Médio

IBOPE - Instituto Brasileiro de Opinião Pública e Estatística

INAF - Indicador de Alfabetismo Funcional

MAPA - Ministério da Agricultura, Pecuária e Abastecimento

MERCOSUL - Mercado Comum do Sul

MS - Ministério da Saúde

POF - Pesquisa de Orçamentos Familiares

REALM - Rapid Estimator of Adult Literacy in Medicine

SAEB -Sistema de Avaliação do Ensino Básico

TOFHLA - Test of Functional Health Literacy Assessment

UNESCO - United Nations Educational, Scientific and Cultural

Organization

WRAT - Wide Range Assessment Test 